\title{
Bacteriology and Antibiotic Resistance Pattern in Community Acquired Urinary Tract Infection
}

\begin{abstract}
Extensive use of antibiotics have resulted in development of resistance among most commonly used drugs in community acquired urinary tract infection (UTI). This study was conducted to identify the resistance pattern in community acquired UTI .We collected urine for routine examination and culture from suprapubic urine in all the cases to avoid any contamination. $E$. Coli was the most common organism identified. Among oral antibiotics, there was high degree of resistance to penicillin group and cephalosporin groups. Among parentral antibiotics, all the cephalosporins were variably resistant except cephaperazonesalbactum.
\end{abstract}

Key Words: Antibiotic resistance, Cephalosporins, UTI.

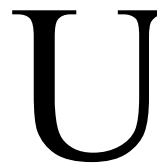
rinary tract infections (UTIs) are often treated with different broad-spectrum antibiotics when one with a narrow spectrum of activity may be appropriate $[1,2]$. Indian Academy of Pediatrics in its revised update on UTI has recommended cephalosporins in oral and parental forms as the first line of therapy [3].

The resistance pattern of community acquired UTI pathogens has not been studied extensively in India [4]. We conducted this study to compare the frequency and drug resistance pattern in uropathogenes isolated from children of $<5 \mathrm{yrs}$ with community acquired UTIs in Jamshedpur, India and to compare the treatment modalities with the recommendation of IAP. Detailed history and complete clinical examination was carried out. The urine specimen was collected in sterile tube through suprapubic approach for investigation. Enrolled cases were grouped under two age groups; upto 2 years, and above 2 years. The Ethics Committee approved the study.

64 children with urine culture positive cases were analyzed. In children less than 2 years, the most common symptoms were fever, diarrhea, and excessive cry. In children between 2 to 5 years of age the most common complaints were fever, dysuria and pain abdomen. E. Coli was the most common organism followed by Klebsiella, Proteus and Pseudomonas. E. coli was most commonly sensitive to nitrofurantoin, followed by levofloxacin, ofloxacin and azithromycin. High degree of resistance was seen for penicillin and cephalosporin groups of drugs. Among parentral antibiotics, $x$ the most sensitive antibiotic was aminoglycosides (amikacin and gentamicin) and high degree of resistance was seen for cephalosporins. Detailed drug sensitivity is shown in Table I.

The evolution of resistance among antibiotic is not a new phenomenon. The high degree of resistance needs to
TABLE I ANTIBIOTIC Resistance PATTERn In CHILDHOOD UTI

\begin{tabular}{lllll}
\hline Antibiotics & $\begin{array}{l}\text { E Coli } \\
(49)\end{array}$ & $\begin{array}{l}\text { Klebsiella } \\
(5)\end{array}$ & $\begin{array}{l}\text { Others } \\
(10)\end{array}$ & $\begin{array}{l}P \text { - } \\
\text { Value }\end{array}$ \\
\hline Amoxicillin & $48(98 \%)$ & $4(80 \%)$ & $10(100 \%)$ & 0.074 \\
Augmentin & $43(88 \%)$ & $3(60 \%)$ & $4(40 \%)$ & 0.002 \\
Cephalexin & $42(85.8 \%)$ & $3(60 \%)$ & $10(100 \%)$ & 0.110 \\
Ciprofloxacin & $31(63.3 \%)$ & $2(40 \%)$ & $6(60 \%)$ & 0.596 \\
Norfloxacin & $41(83.7 \%)$ & $1(20 \%)$ & $7(70 \%)$ & 0.005 \\
Ofloxacin & $13(26.6 \%)$ & 0 & $7(70 \%)$ & 0.112 \\
Septran & $37(75.6 \%)$ & $3(60 \%)$ & $9(90 \%)$ & 0.406 \\
Azithromycin & $14(28.6 \%)$ & 0 & $3(30 \%)$ & 0.373 \\
Levofloxacin & $3(6.2 \%)$ & 0 & $4(40 \%)$ & 0.005 \\
Nitrofurantoin & 0 & $1(20 \%)$ & $4(40 \%)$ & 0.000 \\
Amikacin (iv) & $10(21.4 \%)$ & $0(0 \%)$ & $3(30 \%)$ & 0.396 \\
Ceftazidime (iv) & $30(61.3 \%)$ & $2(40 \%)$ & $9(90 \%)$ & 0.114 \\
Cefuroxime (iv) & $33(67.4 \%)$ & $2(40 \%)$ & $8(80 \%)$ & 0.298 \\
Gentamicin (iv) & $8(16.4 \%)$ & $0(0 \%)$ & $4(40 \%)$ & 0.116 \\
Magnex (iv) & $10(21.4 \%)$ & $0(0 \%)$ & $3(30 \%)$ & 0.396 \\
Imipenem (iv) & $20(41.9 \%)$ & $2(40 \%)$ & $8(80 \%)$ & 0.073 \\
Meropenem (iv) & $19(38.8 \%)$ & $1(20 \%)$ & $7(70 \%)$ & 0.110 \\
Cefotaxime (iv) & $36(73.5 \%)$ & $3(60 \%)$ & $7(70 \%)$ & 0.807 \\
Ceftriaxone (iv) & $36(73.5 \%)$ & $2(40 \%)$ & $9(90 \%)$ & 0.118 \\
Pipzo (iv) & $21(42.2 \%)$ & $0(0 \%)$ & $4(40 \%)$ & 0.173 \\
& & & &
\end{tabular}

be reviewed in the light of our report as cephalosporins are among the highly prescribed drugs over the counter.This adds to inadequate treatment, high degree of resistance and increased cost of treatment [5]

Contributors: All the authors designed, contributed and approved the study.

Funding:Tata Motors; Competing interests: None stated.

RaJiv Sharan, Dhananjay KuMar and B MUKherJeE Tata Motors Hospital, Jamshedpur, Jharkhand, India. rajiv.sharan@tatamotors.com

\section{REFERENCES}

1. Schaeffer AJ. The expanding role of fluoroquinolones. Am J Med. 2002;113:45S-54S.

2. Biswas D, Gupta P, Prasad R, Singh V, Arya M, Kumar A. Choice of antibiotic for empirical therapy of acute cystitis in a setting of high antimicrobial resistance. Indian J Med Sci. 2006,60:53-8.

3. Indian Society of Pediatric Nephrology. Revised Statement on Management of Urinary Tract Infections. Indian Pediatr. 2011;48: 709-17.

4. Gupta V, Yadav A, Joshi RM. Antibiotic resistance pattern in uropathogen. Indian J Med Microbiol. 2002;20:96-8.

5. Costelloe C, Metcalfe C, Lovering A, Mant D, Hay AD. Effect of antibiotic prescribing in primary care on antimicrobial resistance in individual patients: systematic review and meta-analysis. BMJ. 2010;340 doi:10.1136/ bmj.c2096. 